\title{
Entrance Region Flow Heat Transfer in Concentric Annuli with Rotating Inner Wall for Herschel-Bulkley Fluids
}

\author{
Srinivasa Rao Nadiminti and A. Kandasamy \\ Department of Mathematical and Computational Sciences, National Institute of Technology Karnataka, Surathkal, Mangalore, Karnataka - \\ 575025, India
}

\begin{abstract}
A finite difference analysis of the entrance region flow heat transfer of Herschel-Bulkley fluids in concentric annuli with rotating inner wall has been carried out. The analysis is made for simultaneously developing hydrodynamic and thermal boundary layer in concentric annuli with one wall being isothermal and other one being adiabatic. The inner cylinder is assumed to be rotating with a constant angular velocity and the outer cylinder being stationary. A finite difference analysis is used to obtain the velocity distributions, pressure drop and temperature variations along the radial direction. Computational results are obtained for various values of aspect ratio, flow index, Prandtl's number and Herschel-Bulkley number. Comparison of the present results with the results available in literature for various particular cases has been done and found to be in agreement.
\end{abstract}

\section{Introduction}

The study of non-Newtonian laminar flow heat transfer in thermal entrance region of an annuli is of practical importance in engineering applications such as design of compact heat exchangers, axial-flow turbo machinery and polymer processing industries. Very often, optimum conditions are provided by laminar flow operations for keeping low pumping power in proportion to the heat transfer rate. In the nuclear reactor field, this happens when coolant flow rates are reduced. In case of turbulent flow, when heating starts at the duct entrance, the hydrodynamic boundary layers normally are linear near the duct entrance and the transitions to turbulence occurs at some distance downstream from the entrance. Hence, it is necessary to take into consideration the presence of such laminar entrance flow in calculating the heat transfer parameters for a duct in which the fully developed flow is turbulent. Many important industrial fluids are non Newtonian in their flow characteristics and are referred to as rheological fluids. These include blood; various suspensions such as coal water or coal-oil slurries, glues, inks, foods; polymer solutions; paints and many others. The fluid considered here is the Herschel-Bulkley model, which is of 'time independent yield stress' fluid category.

The problem of entrance region flow heat transfer in a concentric annuli for a Newtonian fluid was studied by Coney and El-Shaarawi [1]. Mishra and Kumar [2] studied the flow of the Bingham plastic in the concentric annulus and obtained the results for boundary layer thickness, centre core velocity, pressure drop. Batra and Bigyani Das [3] developed the stress-strain relation for the Casson fluid in the annular space between two coaxial rotating cylinders where the inner cylinder is at rest and outer cylinder is rotating. Maia and Gasparetto [4] applied finite difference method for the Power-law fluid in the annuli and found difference in the entrance geometries. Sayed-Ahmed and Hazem [5] have applied finite difference method to study the laminar flow of a Power-Law fluid in the concentric annuli with rotating inner wall.

The Herschel-Bulkley model represents the empirical combination of Bingham and power-law fluids. The constitutive equation for these fluids is given by Bird et al. [6]as

$$
\tau=\tau_{0}+k\left(\frac{\partial u}{\partial r}\right)^{n}
$$

where $\tau$ is the shear stress, $\tau_{0}$ is the yield stress, $\mathrm{k}$ is the consistency index, $\mathrm{n}$ is the flow index of the model.

Manglik and Fang [7] numerically investigated the flow of non-Newtonian fluids through annuli. Round and $\mathrm{Yu}$ [8] analyzed the developing flows of HerschelBulkley fluids through concentric annuli. Soares et al. [9] has taken up the problem of heat transfer in a fully developed flow of Herschel-Bulkley materials through annular spaces, with insulated outer wall and uniform heat flux at inner wall. Kandasamy et al. [10] investigated the entrance region flow of heat transfer in concentric annuli for Herschel Bulkley fluids and presents the velocity distributions, temperature and pressure in the entrance region. Poole and Chhabra [11] reported the results of a systematic numerical investigation of developing laminar pipe flow of yield stress fluids. 
Recently, Kandasamy and Srinivasa Rao [12], [13] have investigated the entrance region flow in concentric annuli with rotating inner wall for Herschel Bulkley and Bingham fluids.

In the present work, the problem of entrance region flow heat transfer of Herschel-Bulkley fluids in concentric annuli has been investigated. The analysis has been carried out under the assumption that the inner cylinder is rotating and the outer cylinder is at rest. With Prandtl's boundary layer assumptions, the equations of conservation of mass, momentum and energy are discretized and solved using linearized implicit finite difference technique. The system of non-linear algebraic equations thus obtained has been solved by the Newton Raphson iterative method for simultaneous non-linear equations. The development of axial velocity profile, radial velocity profile, tangential velocity profile, pressure drop and temperature variations in the entrance region have been determined for different values of nonNewtonian flow characteristics and geometrical parameters. The effects of these on the temperature variations have been discussed.

\section{Formulation of the problem}

The Herschel-Bulkley fluid enters the horizontal concentric annuli with inner and outer radii $R_{1}$ and $R_{2}$, respectively, from a large chamber with a uniform flat velocity profile $u_{0}$ along the axial direction $\mathrm{z}$ and with an initial pressure $p_{0}$ and temperature $t_{0}$. The inner cylinder rotates with an angular velocity $\omega$ and the outer cylinder is at rest. The geometry of the problem is shown in Fig.1. The flow is steady, laminar, incompressible, axisymmetric with constant physical properties, having negligible viscous dissipation and no internal heat generation. Moreover, it is assumed that the axial heat diffusion is negligible as compared to the radial diffusion. We consider a cylindrical polar coordinate system with the origin at the inlet section on the central axis of the annulus, the $\mathrm{z}$-axis along the axial direction and the radial direction $r$ perpendicular to the $\mathrm{z}$-axis.

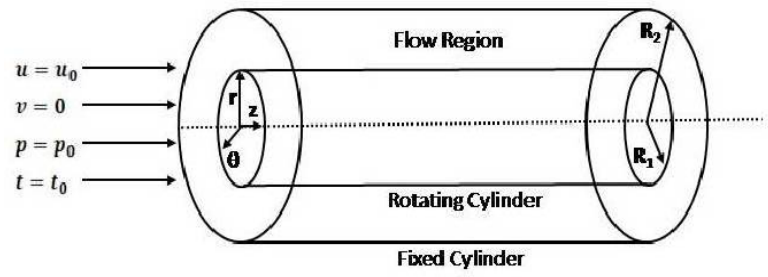

Figure 1. Geometry of the problem.

Under the above assumptions with the usual Prandtl's boundary layer assumptions [14], the governing equations in polar coordinate system $(r, \theta, z)$ for a Herschel-Bulkley fluid in the entrance region are:

$$
\begin{aligned}
& \text { Continuity Equation: } \frac{\partial(r v)}{\partial r}+\frac{\partial(r u)}{\partial z}=0 \\
& \text { r-momentum Equation: } \frac{w^{2}}{r}=\frac{1}{\rho} \frac{\partial p}{\partial r}
\end{aligned}
$$

$\theta$-momentum Equation:

$$
v \frac{\partial w}{\partial r}+u \frac{\partial w}{\partial z}+\frac{v w}{r}=\frac{1}{\rho r^{2}} \frac{\partial}{\partial r}\left(r^{2}\left(\tau_{0}+k\left[r \frac{\partial}{\partial r}\left(\frac{w}{r}\right)\right]^{n}\right)\right)
$$

z-momentum Equation:

$$
v \frac{\partial u}{\partial r}+u \frac{\partial u}{\partial z}=-\frac{1}{r} \frac{\partial p}{\partial z}+\frac{1}{\rho r} \frac{\partial}{\partial r}\left(r\left[\tau_{0}+k\left(\frac{\partial u}{\partial r}\right)^{n}\right]\right)
$$

Energy Equation:

$$
v \frac{\partial t}{\partial r}+u \frac{\partial t}{\partial z}=\alpha\left[\frac{\partial^{2} t}{\partial r^{2}}+\frac{1}{r} \frac{\partial t}{\partial r}\right]
$$

where $\mathrm{u}, \mathrm{v}, \mathrm{w}$ are the velocity components in $\mathrm{z}, \mathrm{r}, \theta$ directions respectively, $\mathrm{t}$ is fluid temperature at any point, $\rho$ is the density of the fluid, $\alpha$ is the thermal diffusivity and $\mathrm{p}$ is the pressure.

The boundary conditions associated with the hydrodynamic part of the problem are given by

for $\mathrm{z} \geq 0$ and $\mathrm{r}=\mathrm{R}_{1}, \mathrm{v}=\mathrm{u}=0$ and $\mathrm{w}=\omega \mathrm{R}_{1}$

for $z \geq 0$ and $r=R_{2}, v=u=w=0$

for $\mathrm{z}=0$ and $\mathrm{R}_{1}<\mathrm{r}<\mathrm{R}_{2}, \mathrm{u}=\mathrm{u}_{0}$

at $\mathrm{z}=0, \mathrm{p}=\mathrm{p}_{0}$

Using the boundary conditions (7), the continuity equation (2) can be expressed in the following integral form:

$$
2 \int_{R_{1}}^{R_{2}} r u d r=\left(R_{2}^{2}-R_{1}^{2}\right) u_{0}
$$

Introducing the following dimensionless variables and parameters

$$
\begin{gathered}
R=\frac{r}{R_{2}}, \mathcal{U}=\frac{u}{u_{0}} \mathbb{P}=\frac{\rho v R_{2}}{\mu_{r}} \mathcal{W}=\frac{w}{\omega R_{1}} N=\frac{R_{1}}{R_{2}} \\
P=\frac{p-p_{0}}{\rho u_{0}^{2}}, \mathcal{Z}=\frac{2 z(1-N)}{R_{2} R e} Y_{h}=\frac{\tau_{0}}{k}\left(\frac{R_{2}}{u_{0}}\right)^{n} \\
T_{a}=\frac{2 \omega^{2} \rho^{2} R_{1}^{2}\left(R_{2}-R_{1}\right)^{3}}{\mu_{r}^{2}\left(R_{2}+R_{1}\right)} \text { here } \mu_{r}=k\left(\frac{\omega R_{1}}{R_{2}}\right)^{n} \\
R e=\frac{2\left(R_{2}-R_{1}\right) u_{0}}{\mu_{r}}, T=\frac{t-t_{0}}{t_{w}-t_{0}} P_{r}=\frac{\mu C_{p}}{K}\left(\frac{\omega R_{2}}{u_{0}}\right)^{1-n}
\end{gathered}
$$

Here $Y_{h}$ is the Hershel-Bulkley number, Re Reynolds number, $T_{a}$ Taylors number, $\mu_{r}$ is known as reference viscosity, $P_{r}$ is the Prandtl's number, $C_{p}$ is the specific heat at constant temperature, $\mathrm{K}$ is the thermal conductivity, $\mathrm{t}_{\mathrm{w}}$ is the isothermal wall temperature and $\mathrm{N}$ is known as aspect ratio of the annulus. 
Equations (2)-(6) and (8) in the dimensionless form are given by

$$
\begin{gathered}
\frac{\partial V}{\partial R}+\frac{V}{R}+\frac{\partial U}{\partial Z}=0 \\
\frac{W^{2}}{R}=\frac{R e^{2}(1-N)}{2(1+N) T_{a}} \frac{\partial P}{\partial R} \\
V \frac{\partial W}{\partial R}+U \frac{\partial W}{\partial Z}+\frac{V W}{R}=\frac{2}{R}\left(\frac{\partial W}{\partial R}-\frac{W}{R}\right)^{n}+ \\
n\left(\frac{\partial W}{\partial R}-\frac{W}{R}\right)^{n-1}\left(\frac{\partial^{2} W}{\partial R^{2}}-\frac{1}{R} \frac{\partial W}{\partial R}+\frac{W}{R^{2}}\right)^{2}+\frac{2 Y_{h}}{R} \\
\frac{\partial U}{\partial R}+U \frac{\partial U}{\partial Z}=-\frac{\partial P}{\partial Z}+\frac{1}{R}\left(\frac{\partial U}{\partial R}\right)^{n}+ \\
n\left(\frac{\partial U}{\partial R}\right)^{n-1} \frac{\partial^{2} U}{\partial R^{2}}+\frac{Y_{h}}{R} \\
V \frac{\partial T}{\partial R}+U \frac{\partial T}{\partial Z}=\frac{1}{P_{r}}\left[\frac{\partial^{2} T}{\partial R^{2}}+\frac{1}{R} \frac{\partial T}{\partial R}\right]
\end{gathered}
$$

and

$$
2 \int_{N}^{1} R U d R=\left(1-N^{2}\right)
$$

The boundary conditions (7) associated with the hydrodynamic part of the problem in the dimensionless form are given by

for $\mathrm{Z} \geq 0$ and $\mathrm{R}=\mathrm{N}, \mathrm{V}=\mathrm{U}=0$ and $\mathrm{W}=1$

for $Z \geq 0$ and $R=1, V=U=W=0$

for $Z=0$ and $N<R<1, U=1$

at $\mathrm{Z}=0, \mathrm{P}=0$

For the thermal part, considering the outer cylinder to be adiabatic and the inner cylinder to be isothermal, the problem has been solved under the following boundary conditions:

for $\mathrm{Z} \geq 0, \mathrm{~T}=1$ at $\mathrm{R}=\mathrm{N}$

for $\mathrm{Z} \geq 0, \frac{\partial T}{\partial R}=0$ at $\mathrm{R}=1$

for $\mathrm{Z}=0$ and $\mathrm{N}<\mathrm{R}<1, \mathrm{~T}=0$

The problem can be similarly analyzed for the case when the inner cylinder is adiabatic and the outer cylinder is isothermal. The boundary conditions for this case will be

for $\mathrm{Z} \geq 0, \mathrm{~T}=1$ at $\mathrm{R}=1$

for $\mathrm{Z} \geq 0, \frac{\partial T}{\partial R}=0$ at $\mathrm{R}=\mathrm{N}$

for $\mathrm{Z}=0$ and $\mathrm{N}<\mathrm{R}<1, \mathrm{~T}=0$

\section{Numerical solutions}

The numerical analysis and the method of solution adopted here can be considered as an indirect extension of the work of Coney and El-Shaarawi [1]. Considering the mesh network of Fig. 2, the following difference representations are made. Here $\Delta R$ and $\Delta Z$ represent the grid size along the radial and axial directions respectively and $\mathrm{m}$ is the number of radial increments in the numerical mesh network.

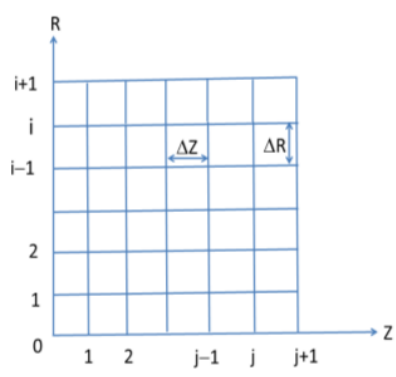

Figure 2.Grid Formation For Finite Difference Representations.

$$
\begin{aligned}
& V_{i+1, j+1}=V_{i, j+1}\left(\frac{N+i \Delta R}{N+(i+1) \Delta R}\right)-\frac{\Delta R}{4 \Delta Z}\left(\frac{2 N+(2 i+1) \Delta R}{N+(i+1) \Delta R}\right) * \\
& \left(U_{i+1, j+1}+U_{i, j+1}-U_{i+1, j}-U_{i, j}\right)
\end{aligned}
$$

$\frac{W_{i, j+1}^{2}}{N+i \Delta R}=\frac{(1-N) R e^{2}}{2 T_{a}(1+N)} \frac{P_{i, j+1}-P_{i-1, j+1}}{\Delta R}$

$V_{i, j}=\left[\frac{W_{i+1, j+1}+W_{i+1, j}-W_{i-1, j}-W_{i-1, j+1}}{4 \Delta R}\right]+$
$U_{i, j}\left[\frac{W_{i, j+1}-W_{i, j}}{\Delta Z}\right]+\frac{V_{i, j} W_{i, j}}{N+i \Delta R}=\frac{2}{N+i \Delta R} *$

$\left[\frac{W_{i+1, j+1}+W_{i+1, j}-W_{i-1, j}-W_{i-1, j+1}}{4 \Delta R}-\frac{W_{i, j}}{N+i \Delta R}\right]^{n}+$

$n\left[\frac{W_{i+1, j+1}+W_{i+1, j}-W_{i-1, j}-W_{i-1, j+1}}{4 \Delta R}-\frac{W_{i, j}}{N+i \Delta R}\right]^{n-1} *$

$\left(\begin{array}{l}\frac{W_{i+1, j+1}+W_{i+1, j}-2 W_{i, j+1}-2 W_{i, j}+W_{i-1, j}+W_{i-1, j+1}}{2(\Delta R)^{2}}- \\ \frac{W_{i+1, j+1}+W_{i+1, j}-W_{i-1, j}-W_{i-1, j+1}}{(N+i \Delta R) 4 \Delta R}+\frac{W_{i, j}}{(N+i \Delta R)^{2}}\end{array}\right)$

$+\frac{2 Y_{h}}{N+i \Delta R}$

$P_{i, j+1}+U_{i-1, j+1}\left[-\frac{\Delta Z}{2 \Delta R} V_{i, j}-\frac{n \Delta Z}{2^{n-1}(\Delta R)^{n+1}}\left(U_{i+1, j+1}-U_{i-1, j+1}\right)^{n-1}\right]+$

$U_{i, j+1}\left[U_{i, j}+\frac{n \Delta Z}{2^{n-2}(\Delta R)^{n+1}}\left(U_{i+1, j+1}-U_{i-1, j+1}\right)^{n-1}\right]+$

$U_{i+1, j+1}\left[\frac{\Delta Z}{2 \Delta R} V_{i, j}-\frac{n \Delta Z}{2^{n-1}(\Delta R)^{n+1}}\left(U_{i+1, j+1}-U_{i-1, j+1}\right)^{n-1}\right]-$

$\frac{\Delta Z}{N+i \Delta R}\left(\frac{U_{i+1, j+1}-U_{i-1, j+1}}{2 \Delta R}\right)^{n}=P_{i, j}+U_{i, j}{ }^{2}+\frac{Y_{h} \Delta Z}{N+i \Delta R}$ 
where $\mathrm{i}=0$ at $\mathrm{R}=\mathrm{N}$ and $\mathrm{i}=\mathrm{m}$ at $\mathrm{R}=1$.

The application of trapezoidal rule to equation (14) gives

$$
\frac{\Delta R}{2}\left(N U_{0, j}+U_{m, j}\right)+\Delta R \sum_{i=1}^{m-1} U_{i, j}(N+i \Delta R)=\left(\frac{1-N^{2}}{2}\right)
$$

The boundary condition (15) gives $U 0, j=U m, j=0$ and the above equation reduces to

$$
\Delta R \sum_{i=1}^{m-1} U_{i, j}(N+i \Delta R)=\left(\frac{1-N^{2}}{2}\right)
$$

The set of difference equations (18) to (23) have been solved by the iterative procedure. Starting at the $\mathrm{j}=0$ column (annulus entrance) and applying equation (20) for $1 \leq i \leq m$-1, we get a system of non-linear algebraic equations. This system has been solved by using Newton Raphson method to obtain the values of the velocity component $\mathrm{W}$ at the second column $\mathrm{j}=1$. Then applying equations (19) and (21) for $1 \leq i \leq m-1$ and equation (22), we get a system of non-linear equations. Again solving this system by Newton-Raphson method, we obtain the values of the velocity component $\mathrm{U}$ and the pressure $\mathrm{P}$ at the second column $\mathrm{j}=1$. Finally, the values of the velocity component $\mathrm{V}$ at the second column $\mathrm{j}=1$ are obtained from equation (18) by Gauss-Jordan method using the known values of $U$. Repeating this procedure, we can advance, column by column, along the axial direction of the annulus until the flow becomes axially and tangentially fully developed. With the values of $\mathrm{V}$ and $\mathrm{U}$ known, the energy equation (13) can be considered as a linear equation in $\mathrm{T}$ with variable coefficients. By using the implicit finite difference technique, the energy equation can be represented as

$$
\begin{aligned}
& T_{i+1, j+1}\left(\frac{V_{i, j+1}+V_{i, j}}{8 \Delta R}-\frac{1}{2 \operatorname{Pr}(\Delta R)^{2}}-\frac{1}{4(N+i \Delta R) \operatorname{Pr} \Delta R}\right)+ \\
& T_{i, j+1}\left(\frac{U_{i, j+1}+U_{i, j}}{2 \Delta Z}+\frac{1}{\operatorname{Pr}(\Delta R)^{2}}\right)+ \\
& T_{i-1, j+1}\left(-\frac{V_{i, j+1}+V_{i, j}}{8 \Delta R}-\frac{1}{2 \operatorname{Pr}(\Delta R)^{2}}+\frac{1}{4(N+i \Delta R) \operatorname{Pr} \Delta R}\right) \\
& =T_{i, j}\left(\frac{U_{i, j+1}+U_{i, j}}{2 \Delta Z}-\frac{1}{\operatorname{Pr}(\Delta R)^{2}}\right)+ \\
& T_{i+1, j}\left(-\frac{V_{i, j+1}+V_{i, j}}{8 \Delta R}+\frac{1}{2 \operatorname{Pr}(\Delta R)^{2}}+\frac{1}{4(N+i \Delta R) \operatorname{Pr} \Delta R}\right)+ \\
& T_{i-1, j}\left(\frac{V_{i, j+1}+V_{i, j}}{8 \Delta R}+\frac{1}{2 \operatorname{Pr}(\Delta R)^{2}}-\frac{1}{4(N+i \Delta R) \operatorname{Pr} \Delta R}\right)
\end{aligned}
$$

Equation (23), with the boundary conditions (16) have been used to obtain the temperature profiles in the annular entrance region. The system of linear equations associated with each column has been solved by Gauss Jordan elimination method.

\section{Results and discussion}

Numerical calculations have been performed for all admissible values of aspect ratio $\mathrm{N}$, flow index $\mathrm{n}$, Prandtl's number and Herschel-Bulkley number. The Prandtl's number has been chosen as 7 . The velocity profiles, pressure drop and temperature distribution along radial direction have been computed for $\mathrm{N}=0.3,0.8$, $\mathrm{n}=0.8,1,1.2$ and $\mathrm{Y}_{\mathrm{h}}=0,10$.

The temperature distribution along radial direction have been plotted for different axial positions with $\mathrm{N}$ $=0.3,0.8, \mathrm{n}=0.8,1,1.2$ and $\mathrm{Y}_{\mathrm{h}}=0,10$. The effects of nonNewtonian characteristics and geometrical parameters on velocity profiles and pressure drop have been extensively discussed elsewhere [12]. Here the temperature distributions during the rotation of the inner wall of the annuli have been shown in Fig. 3-Fig. 8.

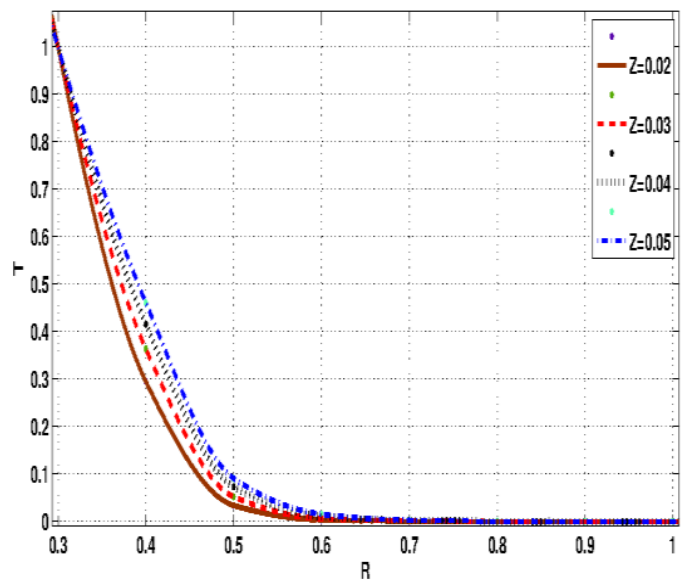

Figure 3. Temperature Distribution for $\mathrm{N}=0.3, \mathrm{n}=0.8, \mathrm{Pr}=7$, $\mathrm{Yh}=0$.

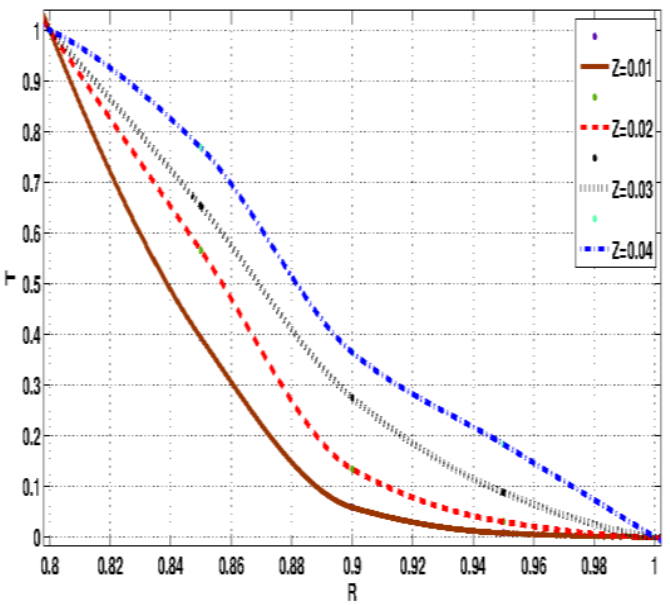

Figure 4. Temperature Distribution for $\mathrm{N}=0.8, \mathrm{n}=0.8, \mathrm{Pr}=7$, $\mathrm{Yh}=10$.

It is observed from the results obtained, that the temperature decreases with increase of Herschel-Bulkley number for a fixed annular width. When the aspect ratio $\mathrm{N}$ increases, it is found that the temperature increases for a fixed Herschel-Bulkley number.

Moreover, with the increase of flow index ' $\mathrm{n}$ ' the temperature decreases for a fixed aspect ratio $\mathrm{N}$ and Herschel-Bulkley number. Also, it is found that with the increase of axial position the temperature also increasing for a fixed aspect ratio N, Herschel-Bulkley number. 
The present results are compared with available results in literature for various particular cases and are found to be in agreement. When the Herschel-Bulkley number $Y_{h}=0$ and $n=1$, our results match with the results corresponded to Newtonian fluid of Coney and ElShaarawi [1]. In the case of stationary cylinders, the results in our analysis are matching with the results of Kandasamy et al. [10]. Also, in the case of non-thermal part these results match with the results of Kandasamy and Srinivasa Rao [12].

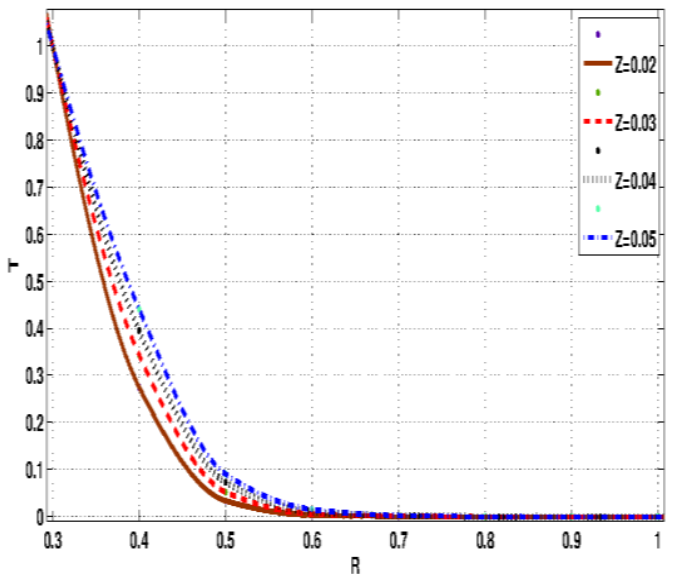

Figure 5. Temperature Distribution for $\mathrm{N}=0.3, \mathrm{n}=1, \mathrm{Pr}=7, \mathrm{Yh}=0$.

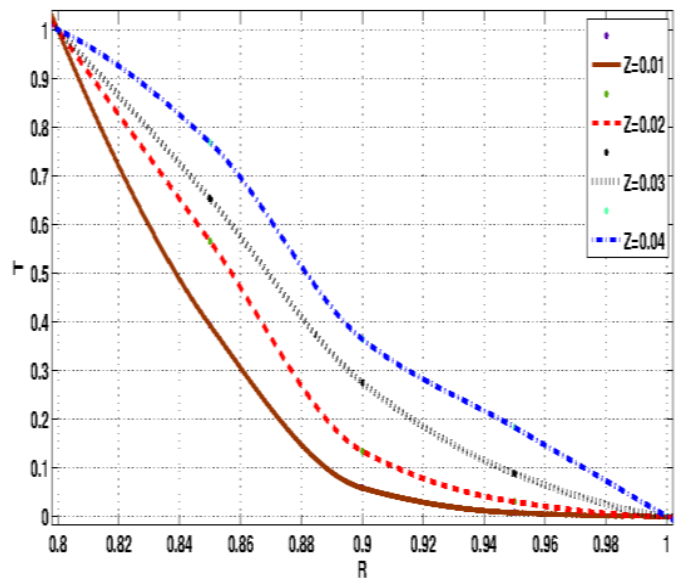

Figure 6. Temperature Distribution for $\mathrm{N}=0.8, \mathrm{n}=1, \operatorname{Pr}=7$, $\mathrm{Yh}=10$.

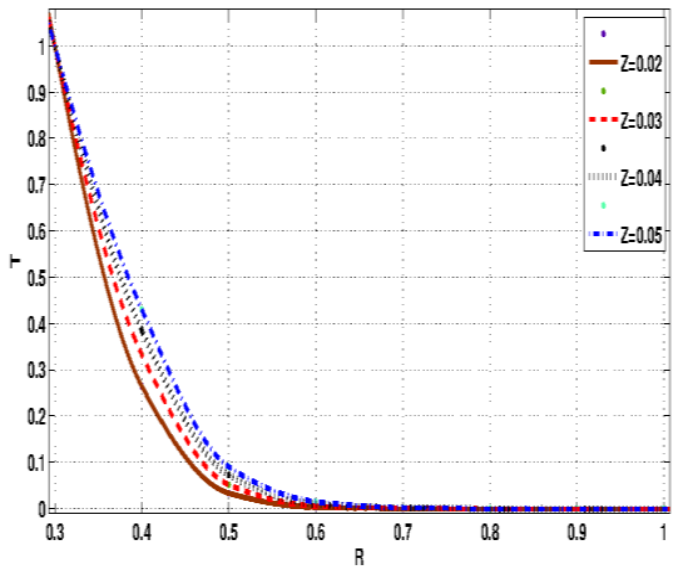

Figure 7. Temperature Distribution for $\mathrm{N}=0.3, \mathrm{n}=1.2, \mathrm{Pr}=7$, $\mathrm{Yh}=0$.

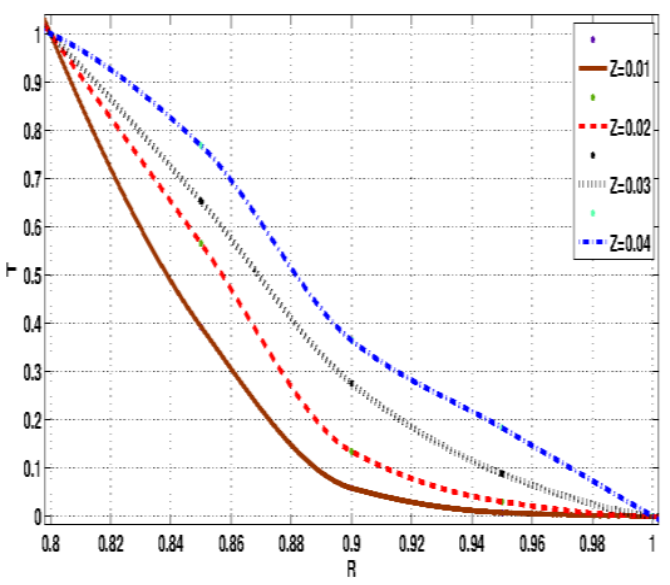

Figure 8. Temperature Distribution for $\mathrm{N}=0.8, \mathrm{n}=1.2, \mathrm{Pr}=7$, $\mathrm{Yh}=10$.

\section{Conclusion}

Numerical results for the entrance region flow heat transfer in concentric annuli with rotating inner wall for Herschel-Bulkley fluids were presented. The effects of the parameters aspect ratio N, Herschel-Bulkley number, flow index and Prandtl's number on the temperature distribution are studied. Numerical calculations have been performed for all admissible values of aspect ratio $\mathrm{N}$, Herschel-Bulkley number, flow index and Prandtl's number. The temperature distribution along radial direction $\mathrm{R}$ have been presented geometrically. The present results are found in agreement with the results corresponding to various particular cases available in literature.

From this study, the following can be concluded.

1. The temperature decreases from the rotating inner wall to the stationary outer wall of the annulus.

2. When increasing the Herschel-Bulkley number, it is observed that the temperature decreases.

3 . With the increase of flow index ' $n$ ', the temperature decreases.

4. When aspect ratio $\mathrm{N}$ increases, it is found that the temperature increases.

5. With the increase of axial position $\mathrm{Z}$, the temperature also increases.

\section{References}

1. J.E.R. Coney, and M.A.I. El-Shaarawi, A contribution to the numerical solution of developing laminar flow in the entrance region of concentric annuli with rotating inner walls, J. Fluid Eng., 96, 333-340 (1974)

2. I. M. Mishra, Surendra Kumar, and $P$ Mishra, Entrance Region Flow of Bingham Plastic Fluids in Concentric Annulus, Indian Journal of Technology, 23, 81-87 (1985)

3. R.L. Batra, and Bigyani Das, Flow of a Casson Fluid between Two Rotating Cylinders, Fluid Dynamic Research, 9, 133-141 (1992)

4. M.C.A. Maia, and C.A. Gasparetto, A numerical solution for entrance region of non-Newtonian flow 
in annuli, Brazilian Journal of Chemical Eng., 20, 201-211 (2003)

5. M.E. Sayed-Ahmed, and Hazem Sharaf-El Din, Entrance region flow of a power-law fluid in concentric annuli with rotating inner wall, International Communications in Heat and Mass Transfer, 33, 654-665 (2006)

6. R.D. Bird, G.C. Dai, and B.J. Yarusso, The rheology and flow of viscoplastic materials, Rev.Chem. Eng., 1, 1-70 (1982)

7. R. Manglik, and P. Fang, Thermal processing of various non-Newtonian fluids in annular ducts, International Journal Heat and Mass Transfer, 45, 803-815 (2002)

8. G.F. Round, and S. Yu, Entrance laminar flows of viscoplastic fluids in concentric annuli, Canadian $J$. Chem. Eng., 71, 642-645 (1993)

9. E.J. Soares, M.F. Naccache, and P.R. Souza Mendes, Heat transfer to Herschel-Bulkley materials in annular flows, Proc. 7th Brazilian Cong. Thermal Sciences, 1146-1151 (1998)
10. A. Kandasamy, K.Karthik, and P.H.Phanidar, Entrance region flow of heat transfer in concentric annuli for Herschel-Bulkley fluids, computational fluid dynamics journal, 16, 103-114 (2007)

11. R.J. Poole, and R.P.Chhabra, Development length requirements for fully developed Laminar pipe flow of yield stress fluids, J. Fluids Eng., 132, 3450134504 (2010)

12. A. Kandasamy and Srinivasa Rao Nadiminti, Entrance region flow in concentric annuli with rotating inner wall for Herschel-Bulkley fluids, Int. Journal of Applied and Comp. Mathematics, 1, 235249 (2015)

13. Srinivasa Rao Nadiminti and A. Kandasamy, Entrance region flow in concentric annuli with rotating inner wall for Bingham fluid, 16th International Conference on Fluid Flow Technologies, Budapest, Hungary, September 1-4, (2015).

14. H. Schlichting, and K. Gersten, Boundary Layer Theory, 8thed., Springer,(2000). 